\title{
Fatigue Life Prediction of an Impeller Based on Fracture Mechanics
}

\author{
Lei Wang* \\ School of Mechanical Engineering \& Automation \\ Northeastern University \\ Shenyang, 110004, China \\ *Corresponding author
}

\begin{abstract}
Welding structure is widely used in impellers fitted on the large centrifugal compressor and the crack propagation life prediction is studied based on the fracture mechanics. First the finite element structure strength analysis is carried on the threedimensional entity model of the impeller, and the dangerous area of the structure is determined. Then based on the mechanical similarity and geometric similarity principles, a simplified twodimensional model of the dangerous area is established. According to the simplified model, the stress intensity factors of two typical kinds of the center crack and the edge crack are calculated and functions of the stress intensity factor changed with the crack length are obtained. Finally, using the mixed mode crack propagation model, the fatigue crack propagation life of the edge crack is estimated by iterative method. The fatigue life prediction method and calculation procedure of the impeller structure is summed up based on the fracture mechanics.
\end{abstract}

Keywords-impeller; life prediction; equivalent model; stress intensity factor; crack propagation

\section{INTRODUCTION}

The impeller is the core component of the high-speed centrifugal compressor rotor. Due to the complexity of the work environment and the structure of the impeller, the fatigue damage often happens. With the increase of diameter and exit width of the impeller, the cracks initiate more easily and gradually extended to fracture, which seriously affect the safe operation of the compressor set. In this paper, the crack propagation life prediction of the first ternary closed impeller for a centrifugal compressor is studied based on the fracture mechanics.

\section{Finite ELEMENT STRUCTURE StRENGTH ANALYSIS}

\section{A. Structure of the Impeller}

The ternary closed impeller is widely used in impellers fitted on the large centrifugal compressor, which the disk, blade and shroud were welded to. The blade is composed by 19 slices which structure is spatial curved surface, and the diameter of the impeller is $1390 \mathrm{~mm}$, as shown in Figure 1.

\section{B. Material and Meshing}

The material of the impeller is FV520B which Elastic modulus, Poisson's ratio, Density and Yield strength are 210GPa, $0.3,7860 \mathrm{~kg} / \mathrm{m}^{3}$ and $1100 \mathrm{MPa}$ respectively. Solid 187

\author{
Tianzhong Sui \\ School of Mechanical Engineering \& Automation \\ Northeastern University \\ Shenyang, 110004, China
}

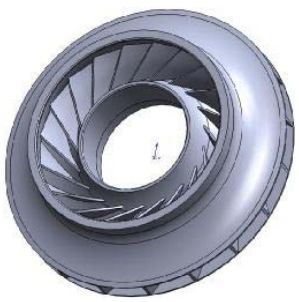

FIGURE I. MODEL OF THE IMPELLER

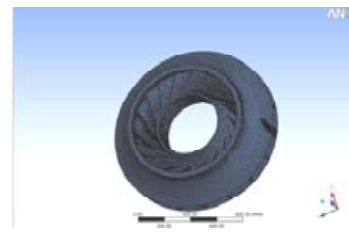

(A) THE WHOLE GRID

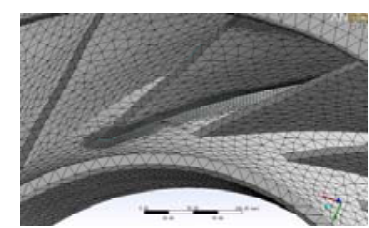

(B) LOCAL GRID REFINEMENT

\section{FIGURE II. MESHING OF THE IMPELLER}

unit is used in meshing, as shown in Figure 2, which consists of 390564 elements and 614542 nodes.

\section{Loading and Constraint}

Fixed displacement is restrained on the shaft hole. The rotate speed is 459.07rad/s, and the pressure field of fluid-solid coupling form which obtained by aerodynamic analysis is applied on the shroud, disk and blade, as shown in Figure 3.

\section{Analysis Results}

The stress distribution of the impeller was shown in Figure 4. It can be seen that the distribution of stress is axisymmetric, and the maximum stress is located in the connection between the blade and the wheel, as shown in Figure 4b.

\section{ESTABLISHMENT OF AN EQUIVALENT MODEL OF THE DANGEROUS AREA}

Based on the mechanical similarity and geometric similarity principles, an equivalent model of the dangerous area is established, as shown in Figure5. The maximum error of the node stress nearby the dangerous area between the equivalent and original models is less than 1.6 percent. 


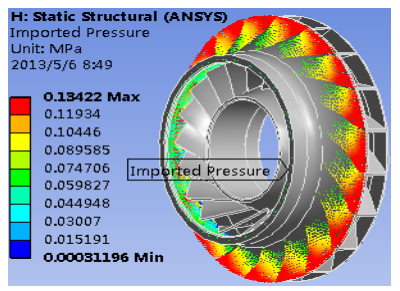

(A) SHROUD LOADING

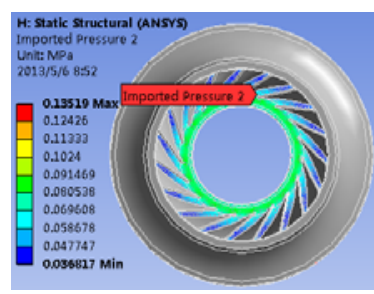

(B) DISK LOADING

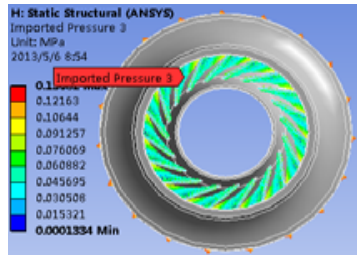

(C) BLADE LOADING

FIGURE III. AERODYNAMIC LOADING

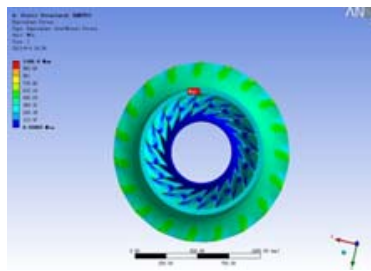

(A)

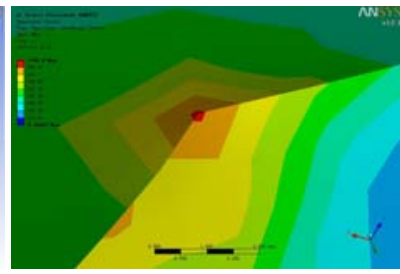

(B)
FIGURE IV. STRESS DISTRIBUTION

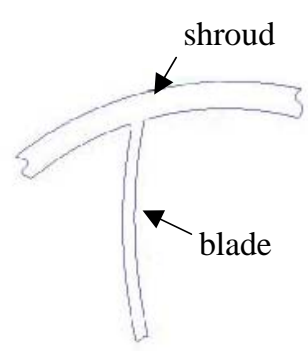

(A) BLADE AND SHROUD

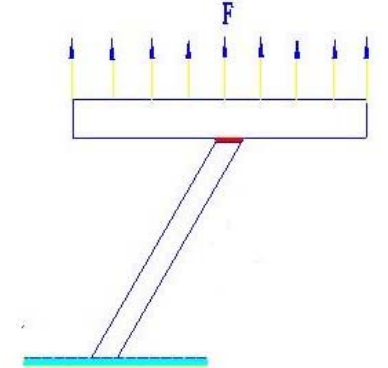

(B)EQUIVALENT MOEDL

\section{FIGURE V. EQUIVALENT MOEDL OF THE DANGEROUS AREA}

\section{DETERMinAtion OF STRESS INTENSITY FACTORS}

Cracks initiation would be easy to occur where the position welded due to the stress concentration, process defects and material defects. The most common forms of crack include the centre crack, edge crack and surface crack, in which the surface cracks are easily found and remedied in the manufacturing process, so the growth behaviors of the centre crack and edge crack were analyzed here.

The stress intensity factor is an important parameter in the analysis of crack propagation, which can determine the stress, strain and displacement near crack tip point. The stress intensity factor must be calculated before estimating the fatigue crack propagation life. In this paper, the stress intensity factor of the dangerous point is calculated by finite element method using the equivalent model.

\section{A. Crack Modeling}

Private welding rod for FV520B is used, and the material around the weld joint is distinguished to the weld zone and thermal response zone as shown in Figure 6.

Then the element type is set to PLANE183, and the crack tip singular elements are created, for example, the center crack is established as shown in Figure 7.

\section{B. Results and Anslysis}

Set the crack length, define the crack path, and solve. Then $\mathrm{K}_{\mathrm{I}}$ and $\mathrm{K}_{\mathrm{II}}$ are obtained under the state of plane stress. The stress intensity factors of the center crack and the edge crack are shown in Table 2 and Table 3 respectively.

Taking the edge crack for example, the fitting curves of the stress intensity factor $\mathrm{K}_{\mathrm{I}}$ and $\mathrm{K}_{\mathrm{II}}$ can be obtained by fitting data in Table 2, which were shown in Formula (1) and (2), Figure 8 and Figure 9.

$$
\begin{aligned}
& y=249.0249+2.0591 t+0.1477 t^{2} \\
& y=86.6066+21.2528 t-2.5694 t^{2}
\end{aligned}
$$

It can be seen that I-II mixed mode crack grows in the early time of the crack propagation. With the increase of the crack length $K_{I}$ is increased and $K_{I I}$ is tend to a fixed value, which lead to the increased ratio of $\mathrm{K}_{\mathrm{I}}$ and the crack turn to open type. It was also found that in the condition of the same crack length the stress intensity factor of edge crack is much higher than the center crack.

\section{Prediction of Fatigue Crack Propagation Life}

\section{A. Cracking Direction and Criterion of Mixed Crack}

For the mixed mode crack, the crack initiation angle is determined by the criterion of maximum stress in tangential direction. The formula is as follow:

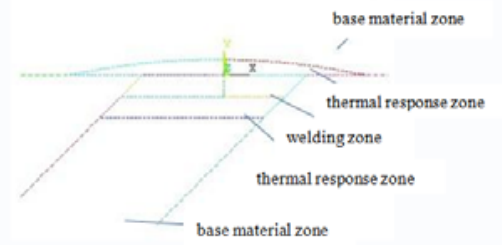

FIGURE VI. MATERIAL AROUND THE WELD JOINT

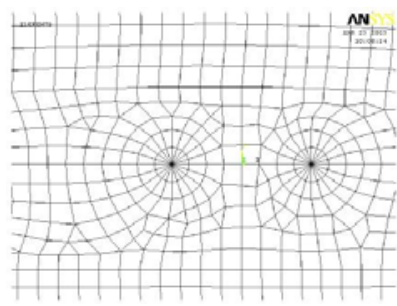

FIGURE VII. MESH OF CRACK TIP 
TABLE I. RESULTS OF THE CENTER-CRACK STRESS INTENSITY FACTORS

\begin{tabular}{|c|c|c|c|c|c|}
\hline \multirow[b]{2}{*}{ No. } & \multirow{2}{*}{$\begin{array}{c}\text { The length of crack } \\
\text { a(mm) }\end{array}$} & \multicolumn{2}{|c|}{ The crack tip A } & \multicolumn{2}{|c|}{ The crack tip B } \\
\hline & & $\begin{array}{c}K_{\mathrm{I}} \\
\left(\mathbf{N} \cdot \mathrm{mm}^{3 / 2}\right)\end{array}$ & $\begin{array}{c}K_{\Pi} \\
\left(\mathbf{N} \cdot \mathbf{m m}^{3 / 2}\right)\end{array}$ & $\begin{array}{c}K_{\mathrm{I}} \\
\left(\mathrm{N} \cdot \mathrm{mm}^{3 / 2}\right)\end{array}$ & $\begin{array}{c}K_{\Pi} \\
\left(\mathbf{N} \cdot \mathbf{m m}^{3 / 2}\right)\end{array}$ \\
\hline 1 & 0.2 & 30.517 & 14.850 & 29.903 & 14.392 \\
\hline 2 & 0.4 & 43.018 & 20.794 & 42.640 & 20.511 \\
\hline 3 & 0.6 & 52.597 & 25.372 & 52.493 & 24.996 \\
\hline 4 & 0.8 & 60.492 & 29.243 & 57.465 & 26.440 \\
\hline 5 & 1.0 & 67.652 & 32.978 & 67.027 & 31.839 \\
\hline 6 & 1.2 & 73.842 & 36.164 & 72.969 & 34.696 \\
\hline 7 & 1.4 & 79.491 & 39.067 & 78.323 & 37.268 \\
\hline 8 & 1.6 & 84.703 & 41.753 & 83.205 & 39.608 \\
\hline 9 & 1.8 & 89.568 & 44.257 & 87.656 & 41.762 \\
\hline 10 & 2.0 & 94.181 & 46.632 & 91.800 & 43.787 \\
\hline
\end{tabular}

TABLE II. RESULTS OF THE EDGE-CRACK STRESS INTENSITY FACTORS

\begin{tabular}{|c|c|c|c|}
\hline No. & The length of crack a(mm) & 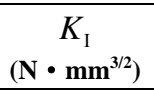 & $\begin{array}{c}K_{\Pi} \\
\left.\mathbf{( N} \cdot \mathbf{m m}^{\mathbf{3} 2}\right)\end{array}$ \\
\hline 1 & 0.2 & 249.443 & 88.905 \\
\hline 2 & 0.4 & 249.901 & 94.540 \\
\hline 3 & 0.6 & 250.260 & 99.493 \\
\hline 4 & 0.8 & 250.680 & 103.040 \\
\hline 5 & 1.0 & 251.390 & 106.150 \\
\hline 6 & 1.2 & 251.740 & 108.890 \\
\hline 7 & 1.4 & 252.160 & 111.442 \\
\hline 8 & 1.6 & 252.649 & 113.815 \\
\hline 9 & 1.8 & 253.180 & 116.080 \\
\hline 10 & 2.0 & 253.783 & 118.232 \\
\hline 11 & 3.0 & 256.638 & 126.291 \\
\hline 12 & 4.0 & 259.432 & 131.147 \\
\hline
\end{tabular}

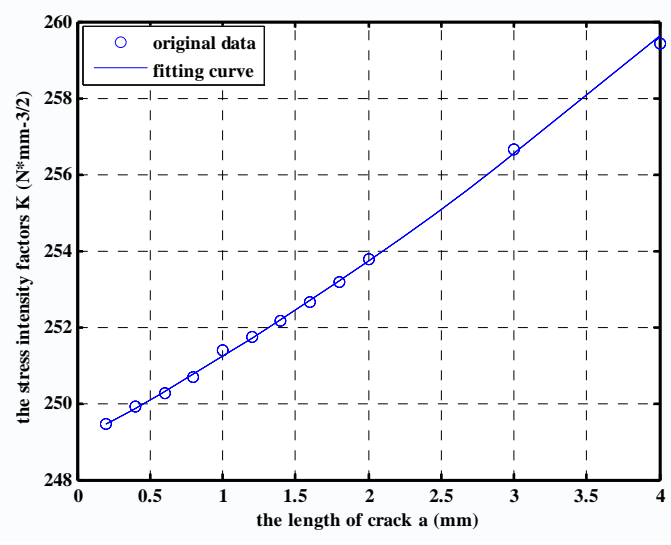

FIGURE VIII. FITTING CURVE OF $K_{\mathrm{I}}$

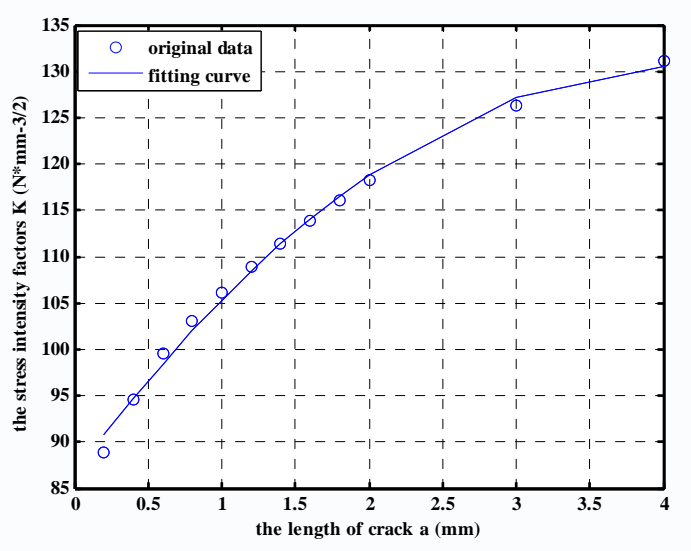

FIGURE IX. FITTING CURVE OF $K_{\text {П }}$ 


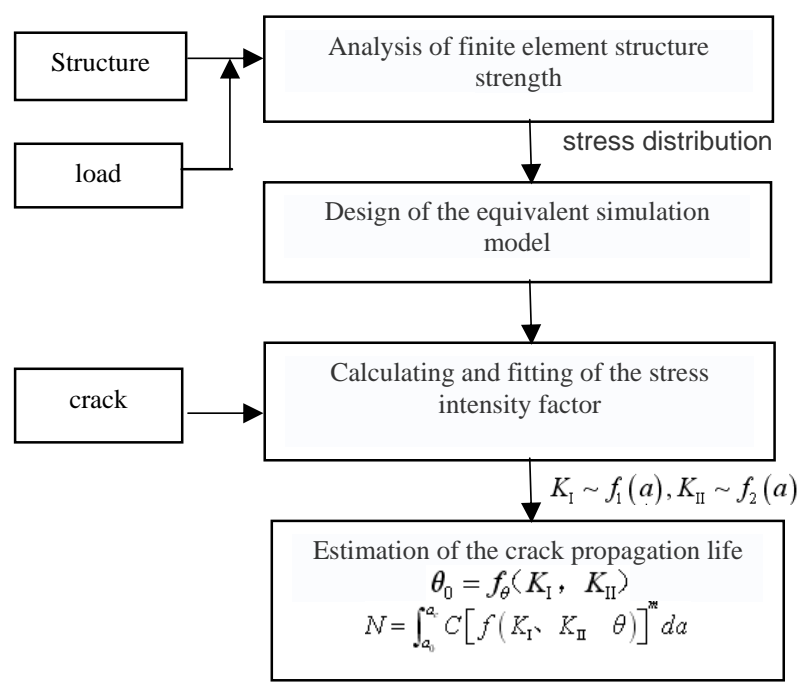

FIGURE X. PREDICTION OF FATIGUE CRACK PROPAGATION LIFE

$$
\theta_{0}=\arccos \frac{3 K_{\Pi}^{2}+\sqrt{K_{I}^{4}+8 K_{\mathrm{I}}^{2} K_{\Pi}^{2}}}{K_{\mathrm{I}}^{2}+9 K_{\Pi}^{2}}
$$

The material failure criterion of the mixed mode crack propagation, namely crack unstable propagation condition, is shown in Formula (4):

$$
\frac{1}{2}\left[K_{\mathrm{I}}\left(1+\cos \theta_{0}\right)-3 K_{\Pi} \sin \theta_{0}\right] \cos \frac{\theta_{0}}{2}=K_{\mathrm{I} c}
$$

where, $K_{\text {Ic }}$ is Fracture toughness of type I crack; $K_{\text {І }}$ and $K_{\text {п }}$ are the stress intensity factors of type I and II crack respectively ; $\theta_{0}$ is the crack initiation angle.

\section{B. Life Prediction Model of the Mixed Mode Fatigue Crack}

Based on the Paris formula (5), in which the combined stress intensity factor $\Delta \mathrm{K}^{*}$ showed in Formula (6) is substituted, the life prediction formula (7) was obtained.

$$
\begin{gathered}
\frac{d a}{d N}=C\left(\Delta K^{*}\right)^{m} \\
\Delta K^{*}=\frac{1}{2}\left(1+\cos \theta_{0}\right) \sqrt{\Delta K_{\mathrm{I}}^{2}-\frac{4 \sin \theta_{0}}{\left(1+\cos \theta_{0}\right)} \Delta K_{\mathrm{I}} \Delta K_{\Pi}+\frac{\left(5-3 \cos \theta_{0}\right)}{\left(1+\cos \theta_{0}\right)} \Delta K_{\Pi}^{2}} \\
N=\int_{a_{0}}^{a_{e}}\left\{C\left[\frac{1}{2}\left(1+\cos \theta_{0}\right) \sqrt{\Delta K_{\mathrm{I}}^{2}-\frac{4 \sin \theta_{0}}{\left(1+\cos \theta_{0}\right)} \Delta K_{\mathrm{I}} \Delta K_{\Pi}+\frac{\left(5-3 \cos \theta_{0}\right)}{\left(1+\cos \theta_{0}\right)} \Delta K_{\Pi}^{2}}\right]^{\mathrm{m}}\right\} d \mathrm{~d}
\end{gathered}
$$

where, ${ }^{a_{0}}$ is the initial crack length; ${ }^{a_{c}}$ is the termination crack length; $\Delta K_{\mathrm{I}}$ and $\Delta K_{\Pi}$ are the amplitudes of stress intensity factor. $\theta_{0}$ is the crack initiation angle; $C$ and $m$ are the material constants.

\section{Prediction of the Fatigue Crack Propagation Life}

Finally, the crack propagation life of the edge crack is calculated .Take $a_{0}=0.02 \mathrm{~mm}, a_{\mathrm{c}}=4 \mathrm{~mm}$,

$$
\begin{aligned}
& \Delta K_{\mathrm{I}}=249.0249+2.0591 a+0.1477 a^{2}, \\
& \Delta K_{\Pi}=86.6066+21.2528 a-2.5694 a^{2} \text { and }
\end{aligned}
$$$$
\theta_{0}=\arccos \frac{3 \Delta K_{\Pi}^{2}+\sqrt{\Delta K_{\mathrm{I}}^{4}+8 \Delta K_{\mathrm{I}}^{2} \Delta K_{\Pi}^{2}}}{\Delta K_{\mathrm{I}}^{2}+9 \Delta K_{\Pi}^{2}}
$$

into Formula (7), and take 0.01 for the integral iteration step distance, it can be obtained that $\mathrm{N}=36736$ times.

\section{Steps for Predicting Fatigue Crack Propagation Life}

The steps of predicting fatigue crack propagation life of the impeller is shown in Figure 10.

\section{CONCLUSIONS}

(1) The structural strength of the impeller is analyzed under the steady-state loading condition. The stress distribution of the welding area between the blades and the wheel are obtained, which is considered as the most dangerous position. It provides the basis for the simplified model.

(2) According to the mechanical and geometry similarity principle, a simplified two-dimensional model of the impeller with the welding area is established, which provided feasible solution for simulating the stress intensity factors.

(3) Combined with fracture mechanics, the stress intensity factors of two typical kinds of the center crack and the edge crack are calculated and functions of the stress intensity factor changed with the crack length are obtained by curve fitting. The simulating results are very valuable to the prediction of crack propagation life.

(4) Using the mixed mode crack propagation model, the fatigue crack propagation life of the impeller is estimated. The fatigue life prediction method and calculation procedure of the impeller structure is summed up based on the fracture mechanics, which are advantageous to the fatigue life prediction of similar structure.

\section{ACKNOWLEDGMENT}

This research was financially supported by the Special Funds for the Major State Basic Research Projects of China (No.2011CB706504) and the fund of basic scientific research for Chinese center university (N120403008).

\section{REFERENCES}

[1] Ramamurti V., Subramani D.A., etal. Free vibration analysis of a turbocharger centrifugal compressor impeller [J], Mechanism and Machine Theory, 1995,30(4):61-628.

[2] Aksoy S., B.Mitlin, H.Borowy. Structural Evaluation and Testing of Swept Compressor Rotor [J]. Journal of Engineering for Gas Turbines and Power, 1994, 116(1):217-222.

[3] Lucjan, Witek. Experimental crack propagation and failure analysis of the first stage compressor blade subjected to vibration [J], Engineering Failure Analysis, 2009, 2(2):1-8. 
[4] Dong C.Y., Lo S.H., Cheumg Y.K. Anisotropic thin plate bending problem by Trefftz boundary collocation method [J], Engineering Analysis with Boundary Element, 2004, 28(9):1017-1024.
[5] Xiao Q.Z., Karihaloo B.L., Liu X.Y. Direct determination of SIF and higher order terms of mixed mode crack by a hybrid element [J], International Journal of Fracture, 2004,125:207-225. 